\title{
Spiking neural network classification for spike train analysis of physiotherapy movements
}

\author{
Fadilla 'Atyka Nor Rashid, Nor Surayahani Suriani \\ Computer Signal, Imaging and Intelligent Research Group, Department of Computer Engineering, \\ Universiti Tun Hussein Onn Malaysia, Malaysia
}

\begin{tabular}{l} 
Article Info \\
\hline Article history: \\
Received Aug 20, 2019 \\
Revised Oct 24, 2019 \\
Accepted Nov 30, 2019 \\
\hline
\end{tabular}

Keywords:

Movements

Recognition

Rehabilitation

Spike trains

Spiking neural networks

\begin{abstract}
Classifying gesture or movements nowadays become a demanding business as the technologies of sensor rose. This has enchanted many researchers to actively investigated widely within the area of computer vision. Rehabilitation exercises is one of the most popular gestures or movements that being worked by the researchers nowadays. Rehab session usually involves experts that monitored the patients but lacking the experts itself made the session become longer and unproductive. This works adopted a dataset from UI-PRMD that assembled from 10 rehabilitation movements. The data has been encoded into spike trains for spike patterns analysis. Next, we tend to train the spike trains into Spiking Neural Networks and resulting into a promising result. However, in future, this method will be tested with other data to validate the performance, also to enhance the success rate of the accuracy.
\end{abstract}

This is an open access article under the $\underline{C C B Y-S A}$ license.

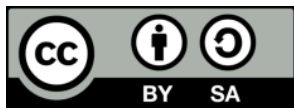

\section{Corresponding Author:}

Nor Surayahani Suriani,

Computer Signal, Imaging and Intelligent Research Group,

Department of Computer Engineering, Faculty of Electrical and Electronic Engineering,

Universiti Tun Hussein Onn Malaysia, Batu Pahat, 86400, Malaysia.

Email: nsuraya @uthm.edu.my

\section{INTRODUCTION}

Physiotherapy, also known as physical therapy, is an ongoing treatment for those who struggling with serious illness such as stroke, Parkinson's, post-surgeries, etc. This therapy is extremely helpful in order for the patients to cope with their daily living tasks due to their movement's impairment. The assessment movements usually will be prescribed according to the patient's condition within their own pace as the movement for a patient may not be equally adequate for others [1]. Furthermore, the patients need to be monitored while performing the movements so that they executed correct movements and their progression can be tracked by medical experts. However, lack of experts makes physiotherapy session delayed and caused discomfort to the patients and the caregivers as well. Hence, technologies take places in assisting the experts conducting the session. Physiotherapy has caught interests of many rese archers in regard to machine learning approaches. Different machine learning algorithms has been utilized for recognizing different types of physiotherapy movements, a lso recognizing different parts of the body.

Recently, neural networks architecture have been a favour for its flexibility and compatibility in a wide range of machine learning applications including computer vision [2]. In the last few decades, the 3rd generation of neural networks, spiking neural networks (SNNs) has been introduced. SNNs are said to be a promising paradigm [3] as it is practically efficient of modelling complex information as they are adequate to represent and integrate different information dimensions (time, space, frequency, phase). Their flexibility and self-organizing manner make it effortless when they are dealing with large volumes 
of data as they are using information representation as trains of spikes [4]. Thus, SNNs intent to sp an the ga p between neuroscience and machine learning which leads in boosting a number of researches with the focus biologically motivated approaches for pattern recognition $[5,6]$.

This paper employs University of Idaho-Physical Rehabilitation Movements Data Set (UI-PRMD) [7] which is a data set of movements performed by patients in physical therapy and rehabilitation programs to classify the types of the movements. This paper proposed spiking neural network (SNN) model to analyse the spike which highly informative for actions recognition to classify the types of the movements. This pa per is structured as follows: Section 2 summarizes related works in classification using UI-PRMD. Section 3 explained the proposed methodology in analysing spike train and classifying by Spiking Neural Network. In section 4, presents experimental results and discussions of the proposed approaches. Finally, conclusions are drawn in Section 5.

\section{PREVIOUS WORK}

Machine learning has been said to be potentially guiding rehabilitation sessions especially in -home rehab sessions as it can improved the ability of recognition and classification. These techniques nowadays are being used extensively in biomedical application [8], for example in recognizing body parts and the types of movements. Previously, Zhu et. al [9] applied K-Nearest Neighbors (KNN) algorithm on rehabilitation prediction and resulting that KNN made significantly better prediction that the Activity of daily living clinical assessment protocol, ADLCAP which being used within the health assessment information system in Canada . Next, they employs Support Vector Machine in [10] to improve upon KNN algorithms as SVM may give more accurate predictions. However, turns out that SVM does not statistically improved over KNN. Muniz et. al [11] compare three different models, SVM, Probabilistic Neural Network (PNN), and Logistic Regression (LR) for discriminating between normal and Parkinson disease persons by monitoring their walking postures. PNN seems performed better than SVM and LR as PNN showed high performance indexes in classifying ground reaction force of normal and Parkinson subjects. While Patsadu et. al [12] compared four approaches for pose classification, Backpropagation Neural Network (BNN), SVM, Decision Tree, and Naïve Bayes. This resulting in BNN and SVM protrude over the other approaches. On the contrary, Neural Networks (NN) and Support Vector Machine (SVM) are also commonly used for poses and motion recognition. Suriani et. al [13] employs SVM to identify a person state whether they in a normal or anomaly movement for fall detection while doing home-based rehabilitation exercises. On the other hand, there are limitations in speed and size for SVM classification. Ciresan et al. used Convolutional Neural Networks (CNN) [14], while Toshev et al. implement Deep Neural Networks (DNN) to recognize human poses and activities [15]. Du et. al divided human skeleton into five segments and used each of the parts to train a hierarchical recurrent neural networks [16].

DNNs are said as a historically brain-inspired but the fundamental are differences in structure, neural computations, and learning rule compare to the brain. The most important difference is the way of the information propagates between their units. Neurons communicate to each other in a neural circuit by sending spike trains which sparse in time so that they have high information content. Spiking Neural Networks (SNNs) are thus more biologically and realistic than DNNs. SNNs also are more hardware friendly and energy-efficient. SNNs shown a promising performance in number of pattern recognition tasks. The architecture consists of spiking neurons where the spike trains communicate with each other through synaptic connections to stimulate the corresponding system. Within the learning from data, the synaptic connections are modified to reflect more precisely the timing of the data from the sensory inputs.

Presently, artificial sensors do not generate spikes as the primary signal instead they are providing us with an electrical analog or digital output encoding its measured value. Thus, various spike encoding method being introduced [17]. SNN has been widely implemented in various area such speech recognition technology as speech signals were converted into spike trains signatures where they are able to differentiate the speech signals that representing different words [18]. In [19], they equipped spike trains for recognition of object and handwritten character and classified with SVM. Next, [20,21] converting analog signals of SA-I mechanoreceptors in human skin into spike trains by an Izhikevich'model and achieved to classify naturalistic textures with an accuracy of 97\%. Hence, they found that SNN exhibit good reliability with strong generalization power of spiking neurons.

\section{RESEARCH METHODOLOGY}

\subsection{Spike train}

SNN represents a special class of artificial neural network (ANN) [22] where ANN have been well developed and had lot of successful application. SNN, as the third generation of neural network [19] also 
to be said as having the potential on solving problem in bio-related stimulation. This neuron models propagates by sequences of spikes or action potentials which conveying information to each other in a membrane potential. The sequences of spikes, or also to be called spike trains, consisting of $n$ spikes occurring at time $t_{i}$ can be represented mathematically as:

$$
\rho(t)=\sum_{i=1}^{n} \delta\left(t-t_{\mathrm{i}}\right)
$$

Where $\delta$ is the Dirac delta function. In order to quantifying the average responses encoded by a single spike train is to simply add up the spikes over some time interval $T$ :

$$
\frac{1}{T} \int_{0}^{T} \rho(\tau) d \tau
$$

This is occasionally called the "firing rate" or "spike count rate". Next, we define an average firing rate over the trials as below:

$$
\frac{1}{T} \int_{0}^{T}\langle\rho(\tau)\rangle d \tau
$$

Where \langle\rangle denotes the average over trials. We also define a firing rate with finer temporal resolution as defining a firing rate $r(t)$ as:

$$
r(t)=\frac{1}{\Delta t} \int_{t}^{t+\Delta t}\langle\rho(\tau)\rangle d \tau
$$

Which also can be used to define Post-Stimulus Time Histogram (PSTH). However, PSTH always defined by depending on where the time bins are placed. Hence, a nother way to define a firing rate is:

$$
r(t)=\int_{-\infty}^{\infty} \omega(\tau) \rho(t-\tau) d \tau
$$

Where $\omega(\tau)$ is called the filter kernel. Nonetheless, a neuron does not have access to the latter information as it is not causal. Thus, a better model conjointly with causalfilter such as:

$$
\omega(t)=\left[\alpha^{2} \tau \exp (-\alpha \tau)\right]_{+}
$$

Where $[x]_{+}$means $x$ for $x \geq 0$, and 0 otherwise. Consequently, we can fit the firing rate to a simple function of the stimulus attributes.

\subsection{Spiking neural networks}

Spiking neurons and synapse are basically the units of an SNN that interconnecting within them. We use the leaking integrate and fire (LIF) [23] models of neurons for the computational tractability where the evolution of the membrane potential, $V_{m}(t)$ is describe by:

$$
C \frac{d V_{m}(t)}{d t}=g L\left(V_{m}(t)-E_{L}\right)+I(t)
$$

Here $I(t)$ is the total input current, $E_{L}$ is the resting potential, and $C(300 p F)$ and $g L(30 n S)$ model the membrane capacitance and leak conductance, respectively [24]. Once the membrane potential crosses a threshold $\left(V_{m}(t) \geq V_{T}\right)$, it is reset to its resting value $E_{L}$ and remains at that value till the neuron comes out of its refractory period $\left(t_{r e f}=3 m s\right)$. The synapse, with weight $w_{k . l}$ connecting input neuron $k$ to output neuron $l$, transforms the incoming spikes (arriving at times $t_{k}^{1}, t_{k}^{2}, \ldots$ ) into a post-synaptic current $\left(I_{k, l}\right)$, based on the following transformation,

$$
\begin{aligned}
& C_{k}(t)=\sum_{i} \delta\left(t-t_{k}^{i}\right) *\left(e^{-\frac{t}{\tau_{1}}}-e^{-\frac{t}{\tau_{2}}}\right) \\
& I_{k, l}(t)=w_{k, l} \times c_{k}(t)
\end{aligned}
$$

Here, the summed $\delta$ function represents the incoming spike train and the double decaying exponentials with $\tau_{1}=5 \mathrm{~ms}$ and $\tau_{2}=1.25 \mathrm{~ms}$ represent the synaptic kernel [25]. 


\section{RESULTS AND ANALYSIS}

\subsection{Experimental setting}

This study tends to propose a classification method for spike train analysis by implementing three-layered spiking neural network. We proposed SNN classification model and documented the achievements. The experiment was carried out using normal CPU and scripted in Matlab \& Simulink library. The computational time is only 30 s per 100 sample. Figure 1 illustrated the general framework of proposed algorithm. Raw data has been parted into 100 for each sample and being generated into firing ra tes to contrast the movements' correctness. Next, we processed the data into spike trains for classification in put. We implement the spike patterns into SNN algorithm for movement classification and tabulate with confusion matrix for validation. Details of each part will be explained in the subtopic below.

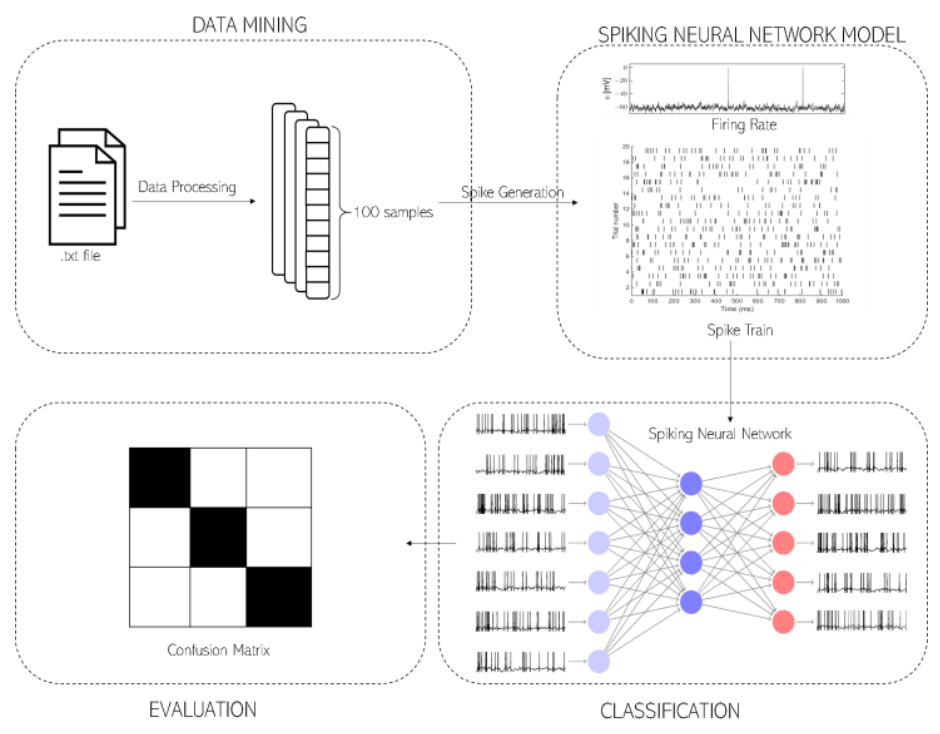

Figure 1. General framework of proposed algorithm

\subsection{Dataset}

This paper employs UI-PRMD dataset [7] which consists 10 rehabilitation movements performed by 10 healthy individuals. Each person performed each movement repetitively 10 times in front of two sensory systems for motion capturing which are Vicon optical tracker and a Kinect camera. The data is collected as positions and angles of the body joints in skeletal models provided by the sensors itself. However, this paper will be focused on Kinect camera angles data with 5 selected rehabilitation movements; Deep Squat, Hurdle Step, Inline Lunge, Sit to Stand, and Standing Shoulder Extension. The results are assessed and discussed in two parts; Spike Train Analysis and SNN Classification.

\subsection{Spike train analysis}

In spike train analysis, data has been parted into 100 frames for each sample and encoded into firing rate and spike train. Firing rate is basically related to the number of spikes generated by a neuron per unit of time. However, in this study, we describe each neuron's firing rate as a function of direction of stimulus motion. Figure 2 shows the comparison of the correctness for the movements which firing spikes generated along $0^{\circ}$ till $360^{\circ}$ orientation direction. The frequencies of firing rates of subjects might be varied as they mostly have different body mass index, but the pattern of the firing rates should be the same to consider the correctness. The incorrect movements might be assembled by incorrect angles of body joints; hence they did not achieve the target angle for perfect movements.

Spike train is built up from a sequence of recorded time at which a neuron fires an action potential. Accumulation of the spike trains in this experiment, leads to a unique pattern for each movement. From Figure 3, each of the movement being recognized has their unique spike patterns which reflect some a spects of neural functioning such as relating neural activity to stimuli, finding a repetitive pattern in a motor discharge and functional interactions between the neurons. Deep Squat and Inline Lunges pattern can be 
defined with similar patterns as physically the movement itself homogenous to each other with the up and down movement direction.

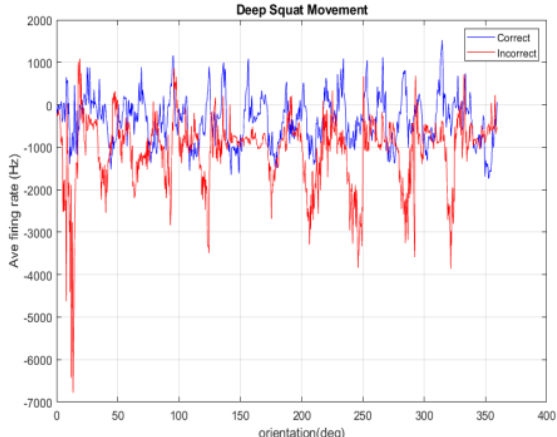

(a)

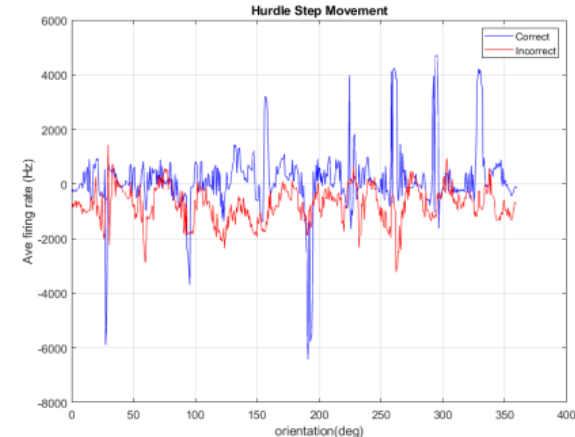

(b)

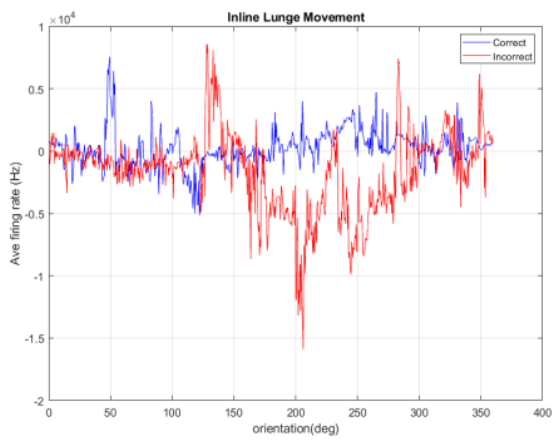

(c)

Figure 2. Example of Comparison of movements' correctness for, (a) Deep squat, (b) Hurdle step, (c) Inline lunges

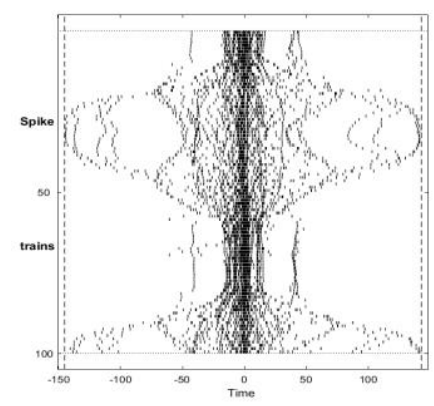

(a)

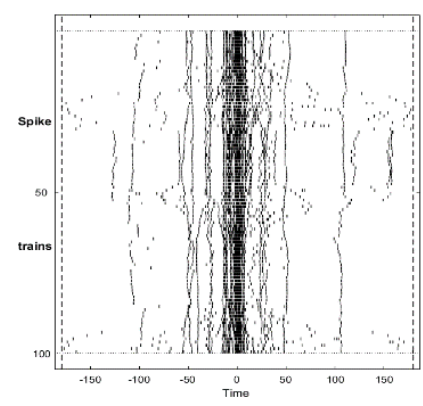

(b)

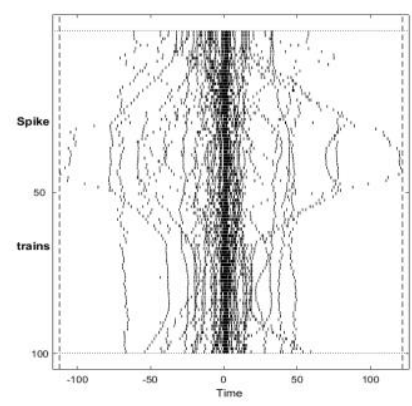

(c)

Figure 3. Example of spike train patterns for, (a) Deep squat, (b) Hurdle step, (c) Inline lunges

\subsection{SNN classification}

In this paper, SNN has been adopted to classify spike trains pattern according to the type of the movements performed. The proposed study is divided into two phases; training and testing. During the training phase, SNN has been trained offline to predict and classify the type of movement from the spike train patterns. For the testing phase, few spike trains were chosen randomly to validate the accuracy 
of the SNN classification. After stimulating the network with spike trains, each neuron starts to specialize to respond to a certain pattern. The ratio of the test and training data is $4: 1$ respectively.

Figure 4 illustrated the accuracy of the classification for the movements based on spike train s analysis classified by SNN. It can be seen from the confusion matrix, SNN achieved 0.71 accuracy and clearly illustrated that the similarity values between the spike's patterns are little bit scattered and Standing Shoulder Extension has the lowest value as it diverged into Deep Squat by 0.29. This discrepancy occurs because of the locational error for the spatial features. Moreover, the patterns of the spike trains are similar to each other so that SNN can't differentiate and classify them accurately. However, in future, we intend to increase the range of the data so that the accuracy might increasing as well.

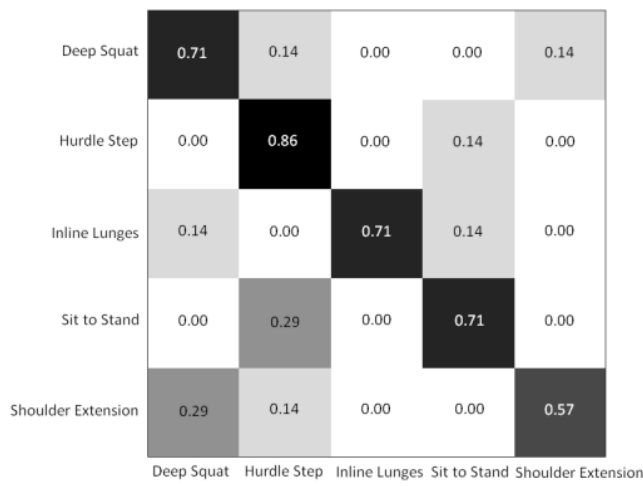

Figure 4. Confusion Matrix for SNN classification accuracy of rehabilitation exercises movement

\section{CONCLUSION}

In this paper, an approach of spiking neural network classification in spike train analysis was proposed. We proposed a simple three-layer spiking neural network that performs the classification of the spike trains to classify the similarity values with the patterns. Our approach achieves $71 \%$ of accuracy which as expected. Thus, this approach is suitable for rehabilitation to be implemented in a stand-alone rehabilitation monitoring session. In future, this method will be tested with other data to validate the performance, also to enhance the success rate of the accuracy. However, we need to revise the algorith $\mathrm{m} \mathrm{s}$ in achieving desired results. Hence, further filtering pre-processing will be implemented to strengthen the proposed method. We will further compare with the other approaches such as SVM, ANN, etc in terms of the classification accuracy. As a conclusion, this proposed method works as our expectation, however, need to be improve for further achievement.

\section{ACKNOWLEDGEMENTS}

This work was supported by the Ministry of Higher Education (MoHE) Malaysia under FRGS grant of Universiti Tun Hussein Onn Malaysia (No. 1584).

\section{REFERENCES}

[1] F. N. Rashid, N. S. Suriani, and A. Nazari, "Kinect-Based Physiotherapy and Assessment: A Comprehensive Review," Indones. J. Electr. Eng. Comput. Sci., vol. 11, no. 3, pp. 1176-1187, 2018.

[2] A. Krizhevsky, I. Sutskever, and G. E. Hinton, "ImageNet Classification with Deep Convolutional Neural Networks," Commun. ACM, vol. 60, no. 6, pp. 84-90, May 2017.

[3] N. Kasabov, K. Dhoble, N. Nuntalid, and G. Indiveri, "Dynamic evolving spiking neural networks for on-line spatio-and spectro-temporal pattern recognition," Neural Networks, vol. 41, pp. 188-201, 2013.

[4] A. Mohemmed, S. Schliebs, S. Matsuda, and N. Kasabov, "Training spiking neural networks to associate spatio-temporal input-output spike patterns," Neurocomputing, vol. 107, pp. 3-10, 2013.

[5] Z. Lin, D. Ma, J. Meng, and L. Chen, "Relative ordering learning in spiking neural network for pattern recognition," Neurocomputing, vol. 275, pp. 94-106, 2018.

[6] I. T. Paz, N. Hernández Gress, and M. González Mendoza, "Pattern Recognition with Spiking Neural Networks BT-Advances in Soft Computing and Its Applications,” pp. 279-288, 2013. 
[7] A. Vakanski, H.-P. Jun, D. Paul, and R. Baker, "A Data Set of Human Body Movements for Physical Rehabilitation Exercises," Data, vol. 3, no. 1, p. 2, Mar. 2018.

[8] P. Lucas, "Bayesian analysis, pattern analysis, and data mining in health care," Curr. Opin. Crit. Care, vol. 10, no. 5, pp. 399-403, 2004.

[9] M. Zhu, W. Chen, J. P. Hirdes, and P. Stolee, "The K-nearest neighbor algorithm predicted rehabilitation potential better than current Clinical Assessment Protocol," J. Clin. Epidemiol., vol. 60, no. 10, pp. 1015-1021, 2007.

[10] M. Zhu, Z. Zhang, J. P. Hirdes, and P. Stolee, "Using machine learning algorithms to guide rehabilitation planning for home care clients," BMC Med. Inform. Decis. Mak., vol. 7, no, 1, p. 41, Dec. 2007.

[11] A. M. S. Muniz et al., "Comparison among probabilistic neural network, support vector machine and logistic regression for evaluating the effect of subthalamic stimulation in Parkinson disease on ground reaction force during gait," J. Biomech., vol. 43, no. 4, pp. 720-726, 2010.

[12] O. Patsadu, C. Nukoolkit, and B. Watanapa, "Human gesture recognition using Kinect camera," in 2012 Ninth International Conference on Computer Science and Software Engineering (JCSSE), pp. 28-32, 2012.

[13] N. S. Suriani, "Fall Detection Using Visual Cortex Bio-inspired Model for Home-Based Physiotherapy System BT-Advances in Machine Learning and Signal Processing," pp. 47-57, 2016.

[14] D. C. Ciresan, U. Meier, J. Masci, L. M. Gambardella, and J. Schmidhuber, "Flexible, High Performance Convolutional Neural Networks for Image Classification," in Proceedings of the Twenty-Second International Joint Conference on Artificial Intelligence-Volume Volume Two, pp. 1237-1242, 2011.

[15] A. Toshev and C. Szegedy, "DeepPose: Human Pose Estimation via Deep Neural Networks," in Proceedings of the 2014 IEEE Conference on Computer Vision and Pattern Recognition, pp. 1653-1660, 2014.

[16] Y. Du, W. Wang, and L. Wang, "Hierarchical recurrent neural network for skeleton based action recognition," in 2015 IEEE Conference on Computer Vision and Pattern Recognition (CVPR), pp. 1110-1118, 2015.

[17] W. Gerstner and W. M, "Spiking Neuron Models: Single Neurons, Populations, Plasticity," Cambridge University Press, 2002.

[18] A. Tavanaei and A. S. Maida, "A spiking network that learns to extract spike signatures from speech signals," Neurocomputing, vol. 240, pp. 191-199, 2017.

[19] P. B. S. G. S. C. A. . A. Khurshid, "Object and Character Recognition Using Spiking Neural Network," in Proceeding Materials Today, vol. 5, no. 1, pp. 360-366, 2018.

[20] U. B. Rongala, A. Mazzoni and C. M. Oddo, "Neuromorphic Artificial Touch for Categorization of Naturalistic Textures," in IEEE Transactions on Neural Networks and Learning Systems, vol. 28, no. 4, pp. 819-829, April 2017.

[21] G. Spigler, C. M. Oddo, and M. C. Carrozza, "Soft-neuromorphic artificial touch for applications in neuro-robotics," in 2012 4th IEEE RAS \& EMBS International Conference on Biomedical Robotics and Biomechatronics (BioRob), pp. 1913-1918, 2012.

[22] F. Ponulak and A. Kasinski, "Introduction to spiking neural networks: Information processing, learning and applications," Acta Neurobiol. Exp. (Wars)., vol. 71, no. 4, p. 409-433, 2011.

[23] S. Dutta, V. Kumar, A. Shukla, N. R. Mohapatra, and U. Ganguly, "Leaky Integrate and Fire Neuron by Charge-Discharge Dynamics in Floating-Body MOSFET," Sci. Rep., vol. 7, no. 1, p. 8257, Aug. 2017.

[24] F. Ponulak and A. Kasiński, "Supervised Learning in Spiking Neural Networks with ReSuMe: Sequence Learning, Classification, and Spike Shifting," Neural Comput., vol. 22, no. 2, pp. 467-510, Oct. 2009.

[25] P. Dayan and L. F. Abbott, "Theoretical Neuroscience: Computational and Mathematical Modeling of Neural Systems,” The MIT Press, 2005.

\section{BIOGRAPHIES OF AUTHORS}
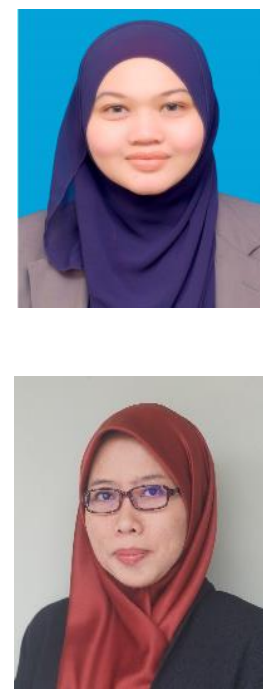

Fadilla Atyka Nor Rashid was born in Johor, Malaysia in 1991. She is a Degree Holder of Computer Network and Security from Universiti Teknologi Malaysia and a master's degree of Telecommunication Engineering from Universiti Teknologi Mara. She is currently a $\mathrm{PhD}$ student in Computer Engineering Department at Universiti Tun Hussein Onn Malaysia. Her area of interests and research are in the computer vision, machine learning, and artificial intelligence. Her main research area is deep learning classification approaches on spike train analysis for physiotherapy movements. She has presented papers in conferences and published a paper in IAES Core journal.

Nor Surayahani Suriani is Senior Lecturer in Universiti Tun Hussein Onn Malaysia (UTHM). She received her $\mathrm{PhD}$ in Electronics and Computer System from Universiti Kebangasaan Malaysia (UKM) in 2015 and finished her Master (Universiti Teknologi Malaysia) and Bac. Eng. (Universiti Putra Malaysia) in Electronics and Communication in 2007 and 2003, respectively. Her research interest focuses on computer vision, image processing and bioinspired visual cortex algorithm development. She has published in Q1 impact factor and Scopus journals mainly in image processing and bio-inspired computer vision areas especially in healthcare and activity monitoring applications. 